\title{
Commentary
}

\section{The Real Cause of Global Warming and its Implications}

\author{
Thomas Allmendinger \\ Glattbrugg (Switzerland)
}

\begin{abstract}
*Corresponding Author: Thomas Allmendinger, Glattbrugg (Switzerland), E mail : inventor@sunrise.ch
\end{abstract}
\begin{abstract}
Referring to a previous treatise of the author where the climate greenhouse theory is refuted in detail [6], first of all the most relevant arguments against this theory are alleged. Apart from some general reasons being apparent even for laymen, additional special reasons are mentioned. They are related to newly discovered absorption phenomena of IR (infrared) radiation at gases [5]. Measuring the temperature enhancements in a tube made from Styrofoam, it was found that any gas adsorbs IR - even noble gases do so -, being warmed up to a limiting temperature which is achieved when the absorption power is equal to the emission power of the warmed gas. It could be theoretically demonstrated that the emission power of a gas is related to the collision frequency of their particles (atoms or molecules) and thus to their size. This explains that no significant differences could be found between argon, pure carbon-dioxide and air. As a consequence, $a$ "greenhouse effect» does not really exist. It would not even take place if the atmosphere were comprised of pure carbon-dioxide! The respective fallacy traces back to early photometric and spectroscopic measurements where - instead of their temperature enhancement - solely the adsorption degree of gases was detected, disregarding the fact that the absorbed IR-light, which is relevant in this case, is re-emitted rather than transformed into heat. On the other hand, these usual methods are too less sensitive for detecting the here described IR-absorption which leads to a temperature enhancement. Hence, the really occurring temperature enhancement of the atmospheric air due to IR-radiation - be it due to the incident solar light, or be it due to the thermal radiation emitted by the Earth surface - has been disregarded so far, while the really not-occurring "greenhouse effect» due to carbon-dioxide has been exclusively promoted.
\end{abstract}

However, this natural absorption effect by the atmosphere cannot be influenced artificially. Instead, the absorption of sunlight by the Earth surface should be focussed, particularly the one of urban areas. It represents the real cause of global warming, since it remarkably governs the temperature of the atmosphere near the ground. Obviously, darker surfaces adsorb more strongly than bright surfaces do, the former being warmed up more intensely. The ratio between reflected and absorbed radiation is called «albedo». But since measurements of the reflected radiation are quite delicate due to the scattering on the surface, a method has been suggested by the author detecting the temperature enhancement of well-defined coloured solid plates, allowing directly determining the solar absorption coefficients directly [8]. Based on this method and its results, referring to [9] several measures at buildings are proposed for mitigating the climate, or at least the microclimates in cities.

In the year 2006, the book and the film of the former US vice president and presidential candidate $A l$ Gore, entitled «An Inconvenient Truth», stirred up the general public. This book and this film did not only emphatically illustrate the consequences of global climate warming but also establish an ostensible explanation alleged by prominent scientists. According to them the carbon-dioxide concentration in the air, arising within the recent period of time, induced a so-called greenhouse effect and was thus responsible for global warming. Al Gore's work was honoured by the Noble Peace Prize and the Documentary Feature Oscar, together with Davis Guggenheim.

At least since then this notion is regarded as the only accurate explication of global warming, being accepted by the large majority of experts and hold by the 'World Climate Council'. It was generally adopted by politicians, culminating in the global climate agreement of Paris 2015 , and pursued by the recent UN climate conference in Bonn, attended by 25'000 participants. But apart from the withdrawal of Donald Trump and the objections from the climate doubters, no scientific objections have been made against this greenhouse theory yet, unanimously accepting it as an indisputable doctrine.

However, it will lead to uncertainties when a large amount of insufficiently informed persons are assessing such a complex subject as atmospheric physics, which would require profound expertise in 
thermodynamics, kinetic gas theory and quantum mechanics, besides practical experience in measuring methods such as IR (infrared) spectroscopy. Moreover, the immense quantity of actual publications impedes the survey, tempting to disregard the basic photometric work of John Tyndall which traces back to the end of the $19^{\text {th }}$ century, and which initiated the greenhouse theory without having an idea of the modern wave concept initiated by Max Planck. Hence the familiarity with this theory usually turns out to be a smattering which relies on an anonymous group of experts.

So it is not surprising that a firm comprehensive description of the greenhouse theory is not available, not even in textbooks such as $[1,2,3]$. It must be picked out from several sources wherein a critical discussion of the diverse variants was not delivered. Instead of scientific arguments, rather consent appears to be decisive being influenced by the majority opinion.

Nevertheless, the greenhouse theory may be briefly described, not least by reference to simplifying articles in the internet which are relevant for the public opinion. It should be realized that it is based on a model, as the name «greenhouse» suggests. Its essential thoughts may be outlined as follows:

The incoming solar light is partly absorbed and partly reflected by the Earth surface. Its absorption degree is given by the complement to the so-called albedo which indicates the reflection degree of the Earth surface, and which mainly depends on its colouring. Due to this absorption, the surface of the Earth is warmed up. Simultaneously, and/or delayed, the warmed Earth surface emits medium-wave IR-radiation (= heat radiation or thermal radiation, wave-length $\lambda=3-50 \mu \mathrm{m}$ ) which is partly absorbed by the atmosphere, due to the greenhouse gases, and partly emitted into Space. Therefore, the assumption is made that any warming-up of the atmosphere is exclusively due to a partial atmospheric absorption of medium-wave IR-radiation by so-called greenhouse gases.

As a consequence, the climate change discussion is usually reduced to the $\mathrm{CO}_{2}$-question. Thereby some aspects of the greenhouse theory should even make the layman suspicious, apart from the difficulty of imaging the Earth atmosphere as a greenhouse. First of all, one should know that the synchronic appearance of two incidents does not prove their mutual interdependency. But such a fallacy is made when the temperature increase of the atmosphere is compared with the increase of its $\mathrm{CO}_{2}$ concentration as it was first published 1982 by the 'Scientific American' [4]. This coincidence is still alleged as a proof for the causal impact of the carbon-dioxide on the atmospheric temperature, disregarding the possibility that another anthropogenic factor which goes along with the industrial progress could be responsible for this effect.

A further doubt should emerge when the low concentration of the carbon-dioxide is taken into account, especially when it is known that its concentration value of $400 \mathrm{ppm}$ is equivalent to 0.04 percent, thus amounting only to approx. a $2500^{\text {th }}$ part of the air. If solely $\mathrm{CO}_{2}$ absorbed thermal radiation leading to a temperature enhancement, it would have to co-warm up the surrounding air, which could not be possible to a considerable extent, due to its large heat capacity.

Besides these trivial objections, an additional objection has to be alleged, concerning the question whether - or to what extent - the absorbed radiation energy of a gas, measured by photometric or spectroscopic methods, is converted into thermal energy inducing a temperature enhancement. It cannot be deduced theoretically but needs empirical investigations. The common atmospheric physics - thus including the greenhouse theory - assumes a priori that this will be entirely the case. But this assumption has never been empirically verified so far. That was the reason for the author's own experiments $[5,6]$ where - instead of the intensity loss of the applied radiation - the temperature enhancements of irradiated gases were measured. They delivered quite surprising results.

\section{The Radiative Warming-up of Gases}

The experiments were carried out with quadratic tubes from Styrofoam $(25$ x 25 x $100 \mathrm{~cm})$ which were covered on both ends by thin plastic foils and equipped with thermometers at three different positions. For minimizing interferences, the thermometer bulbs and the tube walls were mirrored with thin aluminium foils (Fig. 1). The tubes could be filled with different gases from steel cylinders. They were exposed to incident solar light as well as to artificial IR-light whose source was mounted on the top of the tube [5]. Additionally, measurements were made using a hot plate which was positioned on the bottom of the tube and which generated thermal radiation with a larger wave length [6].

In any case, a temperature enhancement was detected leading to a limiting temperature which depended on the intensity of the light source and on the gas type. This phenomenon can be explained 
by the thermal radiation of the gas which increases with increasing temperature up to the extent where the radiated energy is equal to the absorbed one. Using the kinetic gas theory and comparing the results for helium, neon and argon (Fig. 2), a proportionality of the thermal radiation power to the collision frequency of the molecules could be demonstrated, being given by the product of the square root of the absolute gas temperature, the pressure and the size of the molecules. But in particular, no significant difference between pure carbon-dioxide, air and argon could be found.

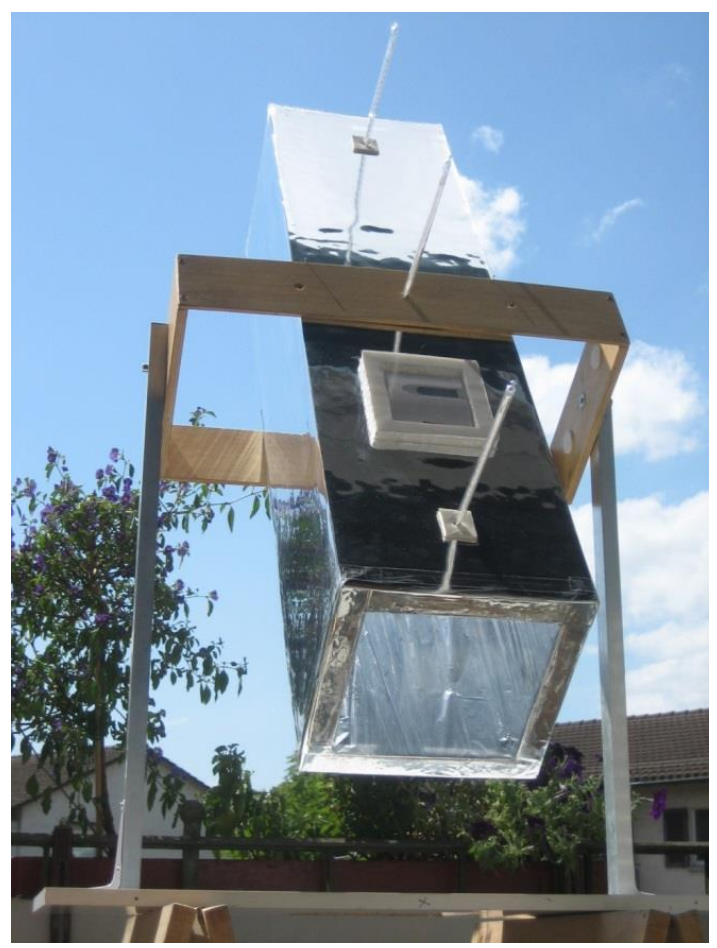

Figure 1. Optimized solar-tube

These results imply that carbon-dioxide has not the slightest influence on the warming-up of the atmosphere. This would be the case even if its concentration were much greater as it really is. Obviously, the absorption which is observed by means of IR-spectroscopy is followed by a radiative emission which happens without affecting the temperature of the gas. On the other hand, another kind of IR-absorption was discovered which affects any gas, leading to a temperature enhancement. However, this kind of absorption is so weak that it cannot be detected by usual IR-spectroscopy.

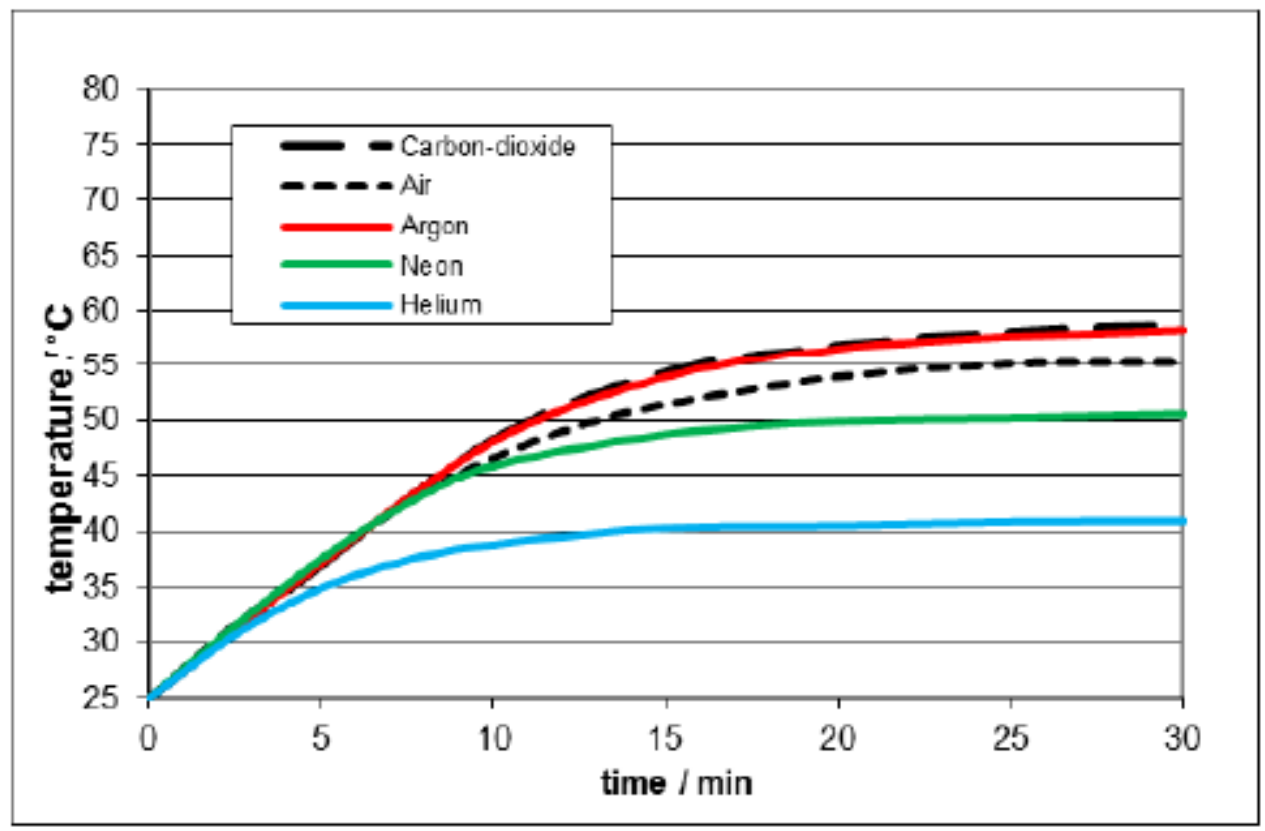

Figure 2. Time-temperature curves of different gases (150 W, medium thermometer position) 
Additionally to these already crucial arguments, a number of further arguments have been alleged in [6] refuting the greenhouse theory and advising to abandon it entirely. Instead, another aspect has to be mentioned providing the real cause of global warming and letting suppose promising measures to mitigate the climate. It concerns the albedo effect of the Earth surface which is correlated to the lightness of colourings. Being particularly obvious in the case of the so called urban heat islands, it has been known for a long time and cannot be denied. Even if there exists an extensive specialized literature propagating 'white roofs' or 'cool roofs', it has been disregarded so far in the public, neglecting it in the climate discussion, and giving precedence to the greenhouse doctrine. Thereby it has been ignored that the albedo, being defined as the intensity ratio between the incident solar light and the light which is reflected by a coloured surface, would act a considerable part - and this even if the greenhouse theory were accurate -, since it governs the temperature of the Earth surface. Hence articles such as the one by H. Hoag suggesting measures "to beat the heat in cities" [7] are still exceptional. The difficult practical determinability of the albedo at materials may be a reason for avoiding the issue. However, the recently published own method [8], where instead of the albedo the complementary solar absorption coefficient is measured, promises a solution to the problem.

\section{The Determination of the Solar Absorption Coefficient}

The here proposed alternative method is based on temperature measurements at the above-mentioned materials, yielding direct values being relevant for the warming-up process and allowing a precise characterization of the materials, while the usual method for detecting the albedo which has been applied so far is based on measurements of the reflected but scattered radiation, delivering solely indirect values.

Using this method [8], the temperature rise of coloured plates, preferably from aluminium, was measured in the presence of vertically incidental solar light (Fig. 3). The temperatures were measured at regular intervals of 5 minutes using thermometers which were centrally inserted in respective holes. The heating-rate could easily be determined by graphically assessing the initial slope. It turned out to depend not only on the surface colour of the plate but also on its volume-specific heat capacity. Evaluating the time/temperature-plots for different coloured plates, their specific solar absorption coefficients $\beta_{\mathrm{s}}$ could be calculated, delivering the results displayed in Fig. 4. Furthermore, assuming complementarity to 1 , the solar reflection coefficients $\alpha_{\mathrm{s}}=1-\beta_{\mathrm{s}}$ could be easily derived. However, it was proposed to distinguish between the solar reflection coefficient and the albedo, the latter one being related to a white surface, according to the original meaning of the word. Thus the albedo represents a relative value, being 1 for any white surface. As a consequence, according to this proposition the albedo and the solar reflecting coefficient are not equal, the latter one being smaller than the former one.

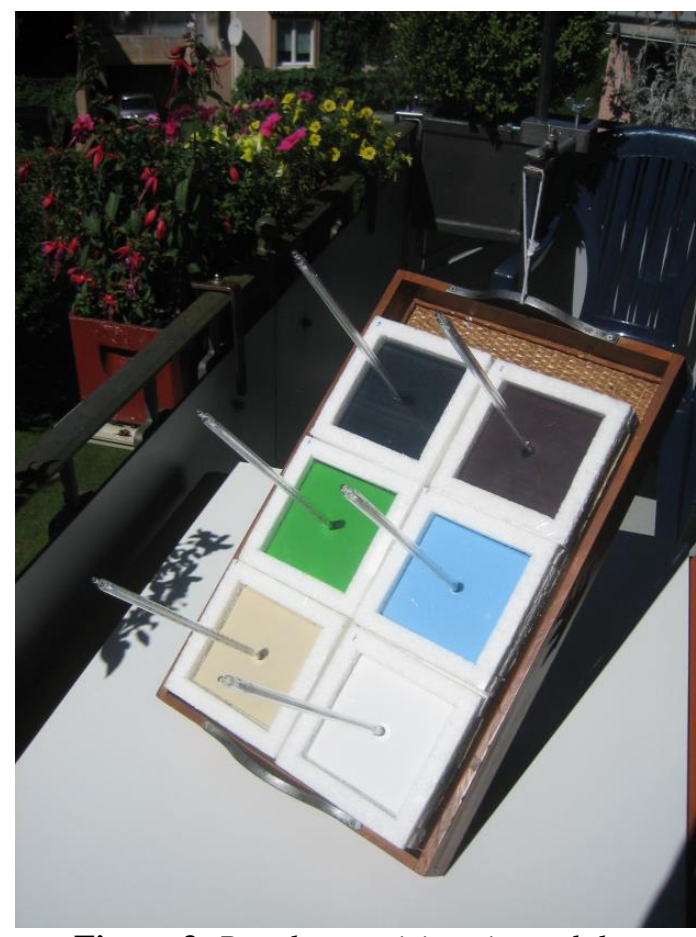

Figure 3. Panel comprising six modules 


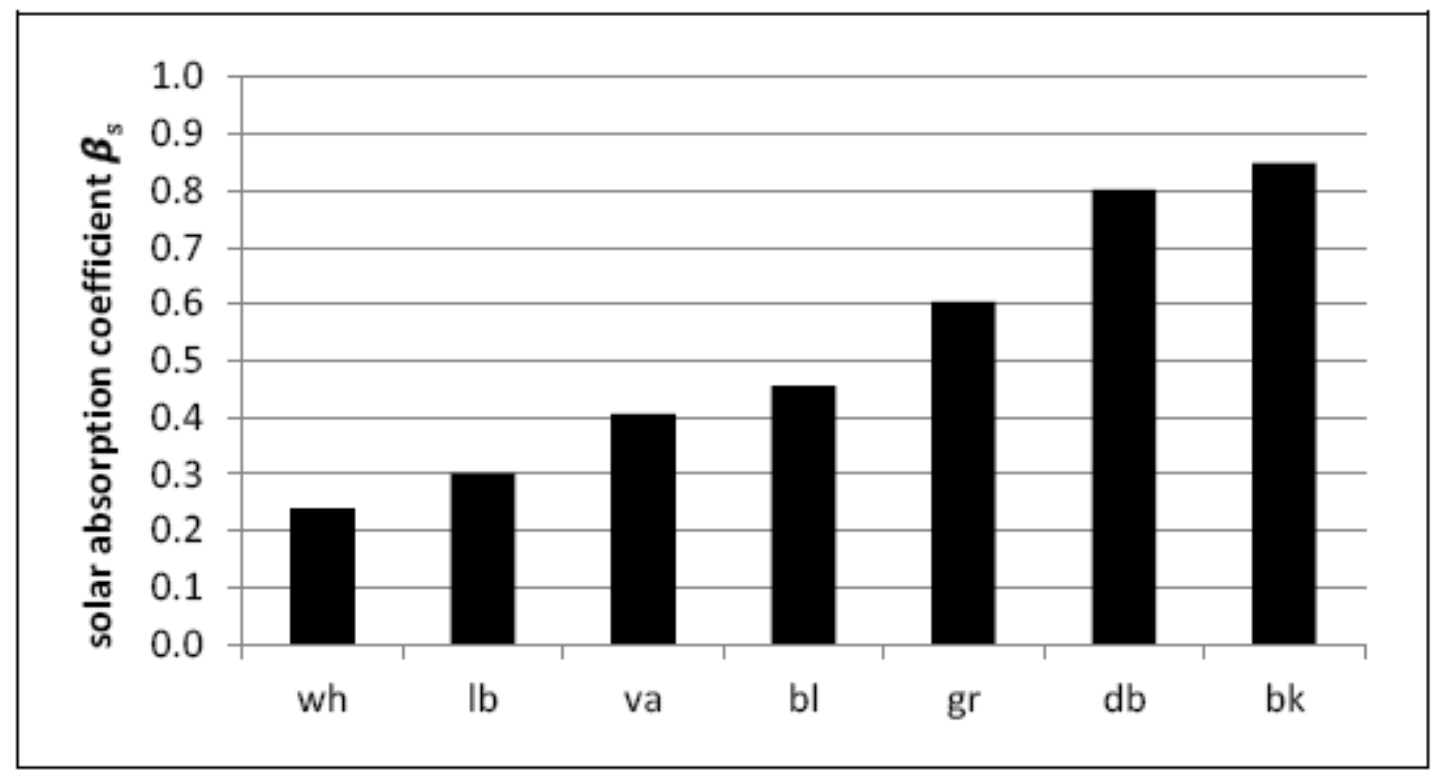

Figure 4. Solar absorption coefficients $\beta$ s on alumina

$(w h=$ white, $l b=$ light brown, $v a=$ vanilla,$b l=$ blue, $g r=$ green, $d b=$ dark brown, $b k=$ black $)$

As it can be anticipated, such plates being exposed to direct sunlight will not be warmed up ad infinitum, but only up to a limiting temperature. Thus the time/temperature-curves will, sooner or later, flatten losing their initially linear character. This phenomenon is already hinted when, instead of aluminium, plates from wood are inserted which exhibit a lower heat capacity implying a quicker warming-up. Obviously, this can be explained with the emission of thermal radiation and an additional heat transfer which effects cooling-down, being temperature dependent and growing till its intensity is equal to the intensity of the absorbed incident solar radiation.

This cooling-down-effect was studied separately in a darkened room, using the same embedding as the one which had been used for the warming-up measurements, but starting from an elevated temperature achieved by preheating the plate in an oven. As expected, the cooling-down rates depended on the heat capacity of the material, leading to lower cooling-down rates for materials with a high heat capacity. But unexpectedly, they did not depend on the surface colour. Moreover, besides thermal radiation, additional heat transfer was involved. As the analysis yielded, the curve-course was exponential, exactly describable with a mathematical formula. Moreover, the heat transfer coefficient could be determined from theseexperimental data, turning out to be in the first approximation independent of the material but dependent on the surrounding atmosphere. However, as it is plausible, the heat conductivity of the material is decisive, too, but scarcely calculable.

The mathematical combination of the respective formula yielded an equation which allowed the mathematical modelling of the time/temperature courses of differently coloured plates. Thereby it turned out that the limiting temperature is independent of the heat capacity, but dependent on the irradiation density, on the solar absorption coefficient, and on the heat transfer coefficient. However, the proportions of the limiting temperatures are equal to the proportions of the warming-up rates and thus of the heat absorption coefficients. This is visualized in the figures 5 and 6 where the temperature courses at differently coloured aluminium-plates, as well as at brick-plates, are plotted. They reveal that the heating-rates of the aluminium-plates are much smaller than those of the brick-plates namely due to the larger heat capacity -, while the limiting temperatures are equal in both cases.

Especially for field measurements, in [8] a simplified method is proposed, too, using a light meter as it is common in photography (Fig. 7). However, it allows solely measuring relative values to a white surface, and thus determining the albedo values but not the solar absorption coefficients. The latter ones can be calculated when the $\beta_{\mathrm{s}}$ value of the white reference plate is known. This was the case here since for both methods the same colour types were applied. As the comparison made in Fig. 8 reveals, the values which were obtained by this method were satisfyingly accurate. 


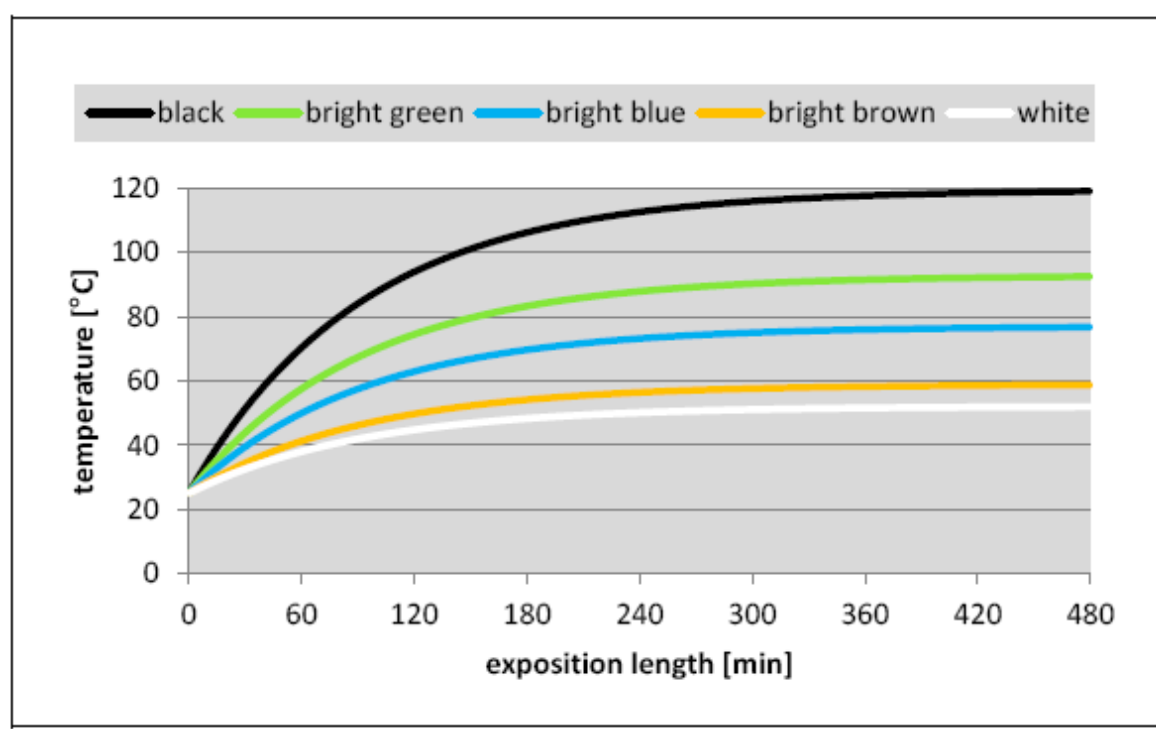

Figure 5. Temperature courses at differently coloured aluminium-plates

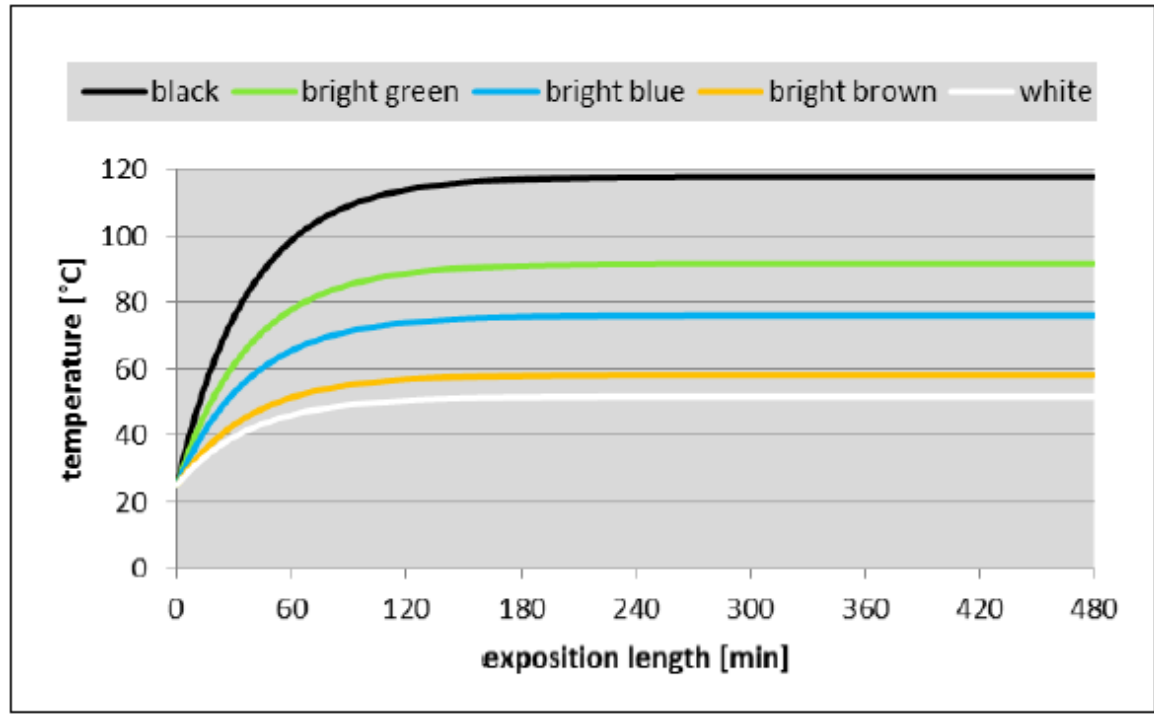

Figure 6. Temperature courses at differently coloured brick-plates

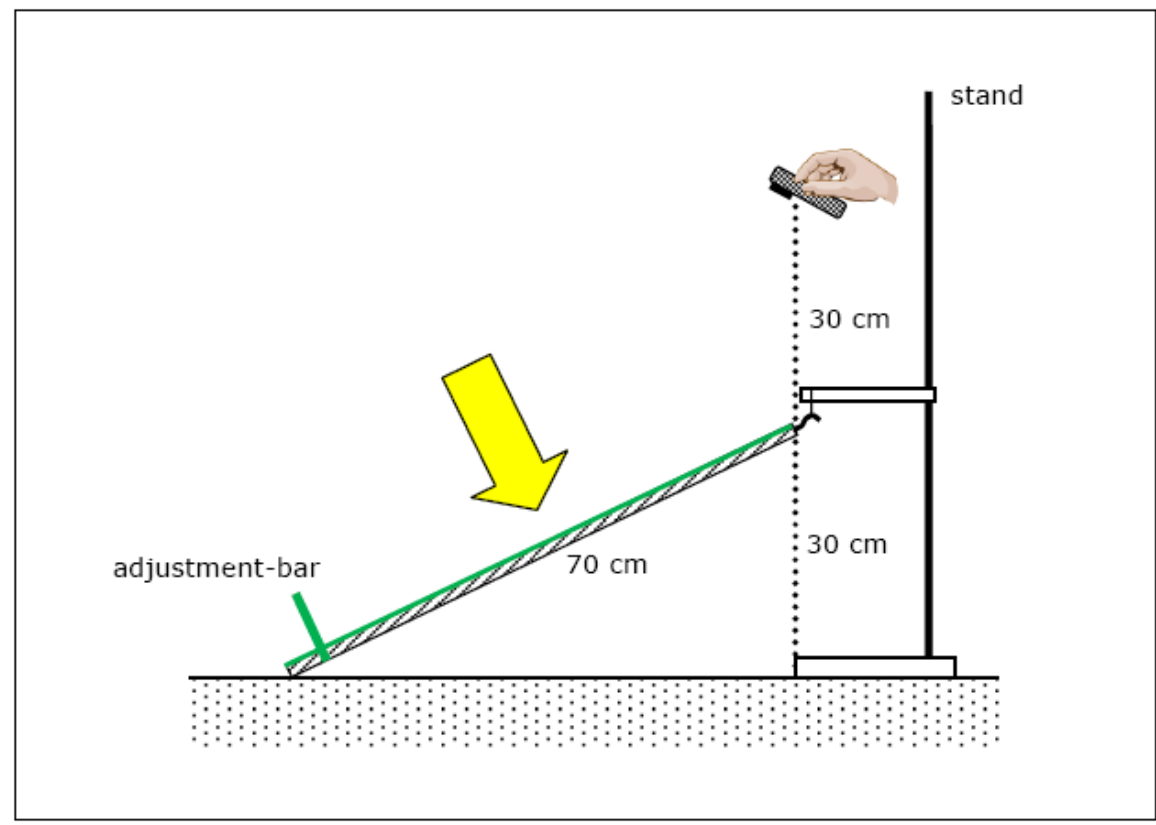

Fig. 7. Assembly for the relative albedo-measurement by a light meter 


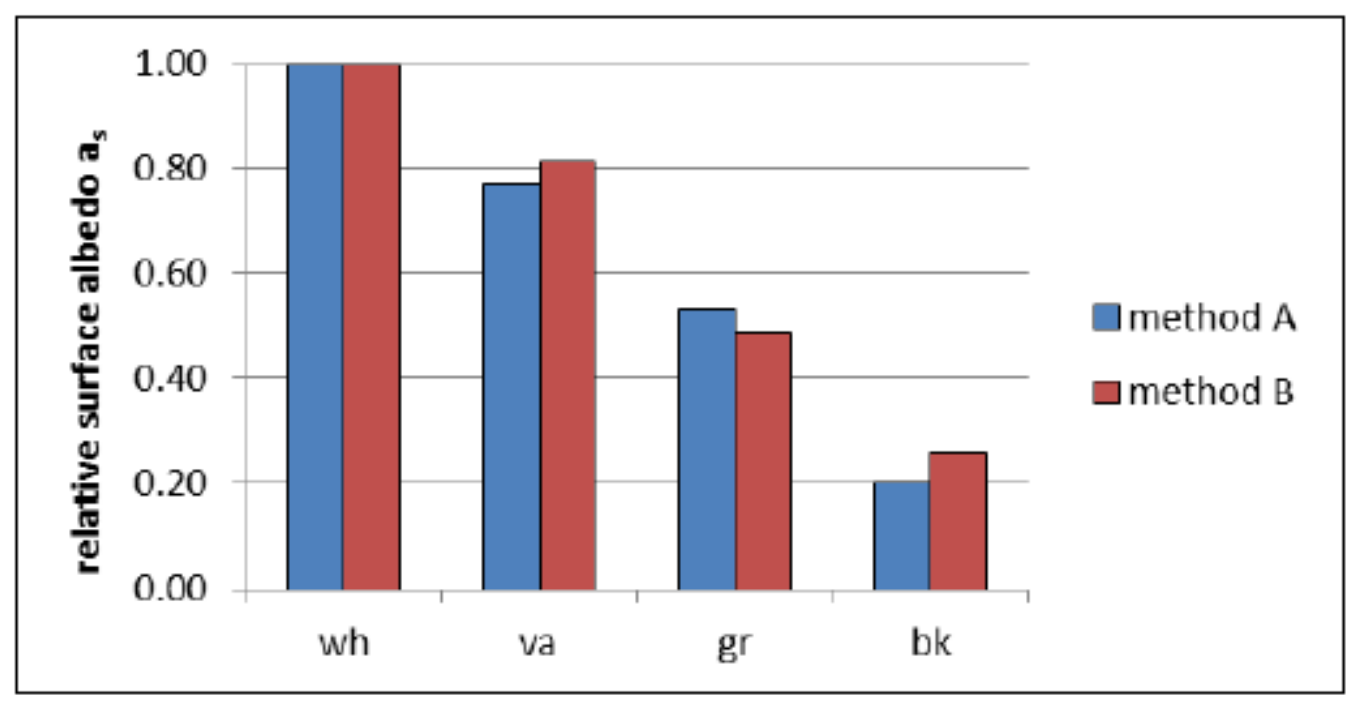

Fig. 8. Method-comparison by means of the albedo-values

(method $A=$ absolute caloric; method $B=$ relative by a light meter)

\section{Promising Measures at Buildings for Mitigating the Micro-Climate}

The implications and results of this method deliver some principal hints which were elucidated in [9] with respect to constructional measures at buildings and urban areas for improving the micro-climate and also the living comfort inside the houses, even if exact forecasts are not possible.

1. The most important influence is due to the surface colouring. As obvious from Fig. 4, (bright) green is not advantageous for applications at buildings since it exhibits a comparatively high solar absorption coefficient. Certainly, it is true that the green colour of plants differs from the artificial green colour which was used in the experiments. It plays an important part in nature since the chlorophyll of the plants is needed for photosynthesis. However, a vegetative temperature regulation by water evaporation is required. Moreover, vegetated areas need maintenance. Hence «green roofs» do not significantly contribute to mitigate the microclimate, in particular when they solely contain lawns. In particular for roofs, rather a lightbrown or straw-yellow colouring is preferred, as shown in Fig. 9, while facades should preferably be white or at least less bright than the roofs. For this purpose, normal colours may be used. Special wondrous paints which lately appeared on the market, promising that black colour takes a white colour effect [10], have not been tested yet using the method described in [9], but they seem to be unnecessary.

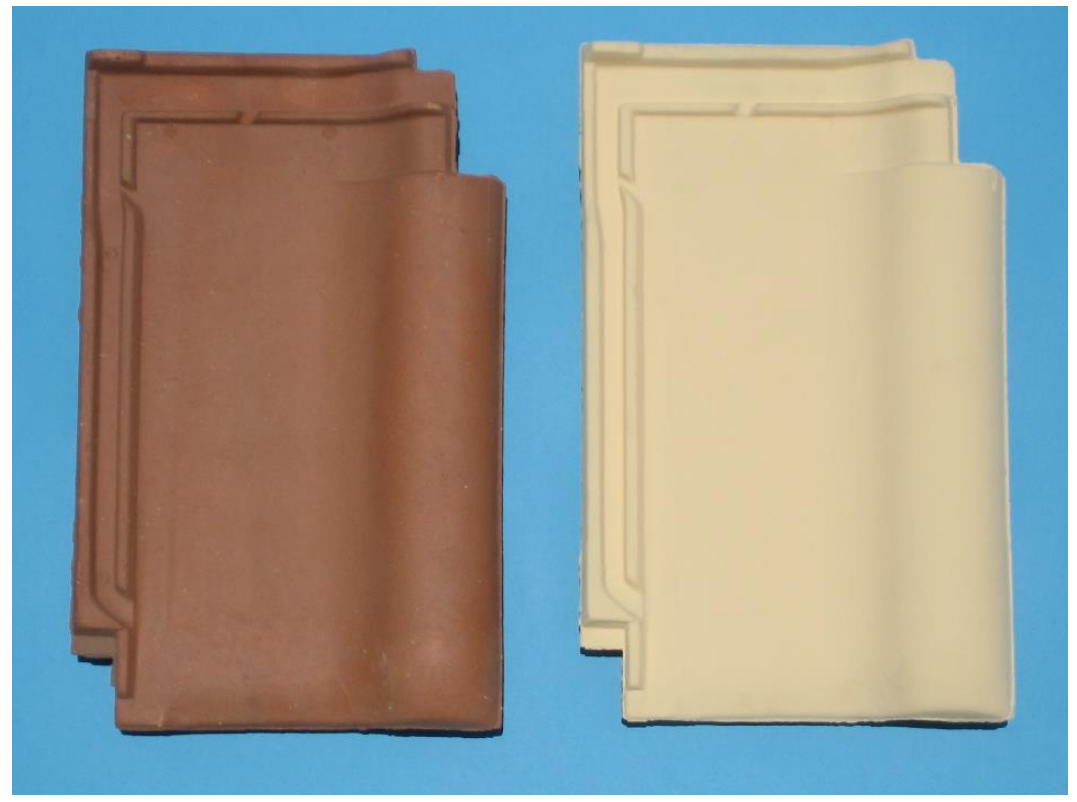

Figure 9. Crude red-brick $(\beta s=0.53)$ and painted one (bright brown, $\beta s=0.30$ ) 
2. The colouring of pavements appears to be problematic since they are exposed to a high pollution and since the normally ensuing grey colour is not too bad. White colouring is out of question since it blinds too much. Light-brown or straw-yellow would be optimal, also from aesthetic reasons, but it seems difficult to realize it replacing asphalt to an acceptable price. So it does not have highest priority. Moreover, a further aspect has to be regarded: the (micro) climate does not solely concern the temperature just above the bottom, i.e. the pavements, but the lowest atmospheric layer as a whole. Hence, the roof-level is of considerable importance even if a pedestrian does not directly feel it. Trees, parks and canopy roads provide shade and contribute to embellish the landscape, emphasizing the emotional component of the climate. However, they do not favour the albedo and thus the radiation budget of a city since their cooling effect is mainly due to evapotranspiration, while the part of energy consumption by photosynthesis is probably low but hard to estimate. In particular, it will not be possible to knock down buildings to a considerable extent, replacing them by trees. Thus the strategy must consist in lightening the cities but not in greening them.

3. However, the best colouring is of limited use when it is destroyed by weathering, as it is normally the case with tiled roofs (Fig. 10). This may be prevented by a protecting layer, preferably by paint. At ancient roofs, this can be made afterwards, too, but then it needs a prior cleaning. Presumably, the weathering of tiles is fortified by acid rain due to a high $\mathrm{CO}_{2^{-}}$ emission in the surroundings. This phenomenon should be a warning signal to the fact that $\mathrm{CO}_{2}$ being emitted in large quantities as a result of fossil fuel combustion, combined with soot, fine dust and other waste gases such as nitrous gases, still represents a considerable source for environmental pollution implying hardly assessable consequences such as acidification of the oceans, presumably leading to a damage of the marine plankton.

4. A further relevant criterion concerns the construction materials since they influence the heating rate because of their heat capacity. Obviously, a high heating rate of walls engenders a high heating rate of the ambient atmosphere. It may be induced either by a dark surface colouring or by a low heat capacity of a wall. A well-insulated wall which exhibits a low heat capacity is solely warmed up at its outer layer since the heat cannot be dissipated within the wall. As a consequence, the ambient atmosphere is heated up while the inside of the house is not affected. For this climatic reason, house walls made from stone are better than those from wood which isolates well but exhibits a low heat capacity. Likewise, additional layers from artificial isolating materials should preferably be attached inside the house walls, and not outside. Moreover, glass facades and windows induce thermal traps, thus being unfavourable.

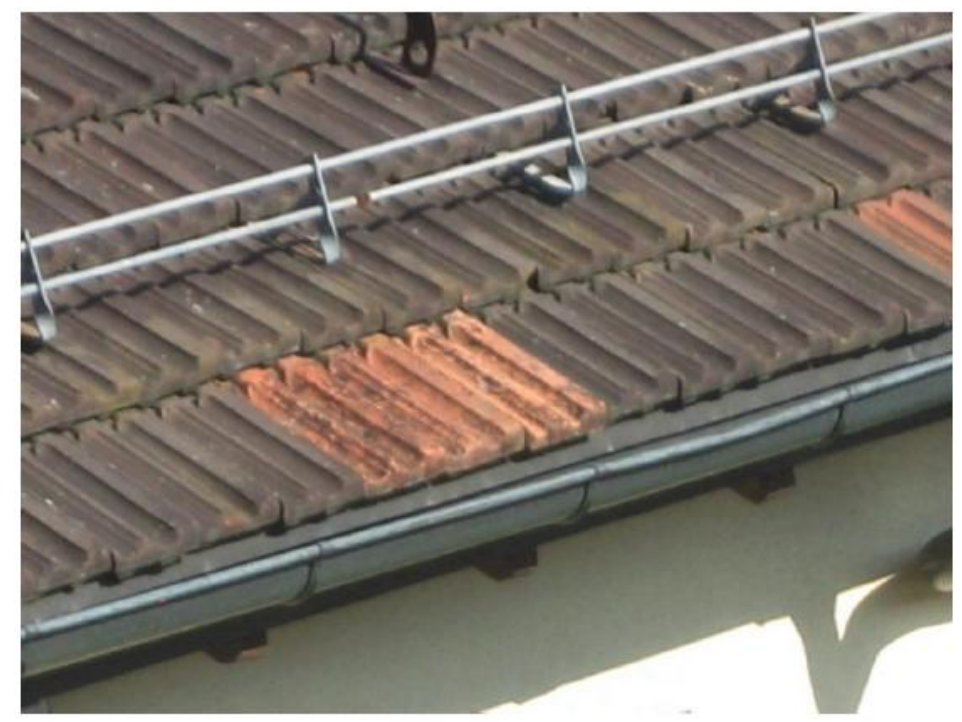

Figure 10. Roof with recently substituted bricks

5. The building dimensions as well as the ambient ground influence radiation characteristics, too. Thereby, considerable radiative interactions between the ground and the facades are to be expected while flat roofs are independent of the other parts. By contrast, gable roofs may partly influence each other, due to the reflected light. In general it can be stated that the solar radiation absorbance of a building group is increased when its macro-roughness is increased. 
It mainly depends on the proportion between altitude and latitude of the buildings. Thus low houses inherently engender an inferior macro-roughness than high houses.

\section{Summery and Conclusions}

The here presented facts reveal that dark colouring of the Earth surface, particularly in cities, represents a special type of environmental pollution. It is high time to realize this, while the greenhouse theory has to be recognized as a phantasm suggesting false and inefficient measures. The only opportunity to mitigate the climate - or at least micro-climates - is given by lightening the Earth surface including the proposed accompanying measures, particularly in cities. Thereby not only the average temperature has to be regarded but also the emergence of temperature gradients, which may be due to local colour differences, and which may thus be responsible for storms. Such a lightening may likewise be the reason to beautify the buildings, in particular the roofs, since the colours which are suited for improving the albedo - or inversely for reducing the solar absorption coefficient - are also suited for embellishing the houses and for improving the living comfort inside the houses. This will also bring an individual benefit to the homeowners. Thereby the applied colours may vary within a specified tinge leaving a margin for the architects.

For achieving a perceptible result, probably hundred millions of roofs would have to be lightened. Obviously, the effect will be intensified at southern locations, but a forecast seems not possible in view of the complexity of the circumstances. However, the efficiency of the taken measures should be monitored. Measures at landscapes are also conceivable but more expensive. Thereby it seems plausible that dark pollutions in the oceans will lead to temperature enhancements of the sea water which may promote the emergence of hurricanes. Altogether, the statement that no climatic greenhouse effect exists does not deliver a blank cheque to an unlimited consumption of fossil fuels since they induce other pollutions, apart from the fact that their reserves are not unlimited.

\section{REFERENCES}

[1] Hartmann D. L. (1994): Global Physical Climatology. Academic Press

[2] Visconti G. (2001): Fundamentals of Physics and Chemistry of the Atmosphere. Springer-Verlag, Berlin, Heidelberg, New York.

[3] Boeker E., van Grondelle R. (2011): Environmental Physics. John Wiley \& Sons Ltd. (3 ${ }^{\text {rd }}$ ed.)

[4] Revelle R. (1982): Carbon Dioxide and World Climate. Scientific American 247/2, 33-41

[5] Allmendinger T. (2016) The thermal behaviour of gases under the influence of infrared-radiation. International Journal of Physical Sciences 11(15): 183-205 http://academicjournals.org/journal/IJPS2016/ article-full-text-pdf/E00ABBF60017

[6] Allmendinger T. (2017) The Refutation of the Climate Greenhouse Theory and a Proposal for a Hopeful Alternative. Environment Pollution and Climate Change 1:2 https://www.omicsonline.org/open-access/therefutation-of-the-climate-greenhouse-theory-and-a-proposal-for-ahopeful-alternative.php?aid=88698

[7] H. Hoag (2015) "How Cities can Beat the Heat", Nature 524, 402-202 http://www.nature.com/news/howcities-can-beat-the heat-1,18228

[8] Allmendinger T. (2016) The solar-reflective characterization of solid opaque materials. International Journal of Science and Technology Educational Research 7(1): 1-17 http://www.academicjournals.org/ journal/IJSTER/article-full-text-pdf/E7435F759158

[9] Allmendinger T. (2017) Measures at Buildings for Mitigating the Microclimate. Environment Pollution and Climate Change 1:3 http://www.academicjournals.org/journal/IJSTER/article-full-text-pdf/E7435F759158

[10] Brady R.F., Detrie T., and Swiler D. (January 1992): When black is white. Paint and Coatings Industry Magazine 